\title{
Hipertiroidismo agudo: um possível caso de síndroma de Marine Lenhart
}

Valter Filipe Moreira, ${ }^{1-2}$ Andreia Teles Ribeiro ${ }^{2}$

\section{RESUMO}

Introdução: O hipertiroidismo (HT) é uma patologia que ocorre devido à síntese e secreção excessiva de hormonas tiroideias pela glândula tiroideia, estimando-se que a sua prevalência a nível mundial se situa entre 1,2 e 1,6\%. A principal etiologia do HT é a doença de Graves (DG), seguida do bócio multinodular (BMN) tóxico. As manifestações clínicas do HT podem variar, desde o quadro assintomático até ao quadro de tempestade tiroideia, uma situação rara de tirotoxicose aguda grave e potencialmente fatal. O síndroma de Marine Lenhart (SML) consiste num quadro de HT em contexto de coexistência de DG e BMN tóxico, sendo uma situação rara que ocorre em 0,8 a $2,7 \%$ dos pacientes com DG.

Descrição do caso: Mulher de 50 anos, com antecedentes pessoais de BMN tóxico não medicado, recorre a consulta de doença aguda por quadro caracterizado por fraqueza extrema com impotência funcional para a marcha, insónia grave, labilidade emocional e irritabilidade associada a palpitações. Foi referenciada ao serviço de urgência (SU) onde foi diagnosticada com HT grave por DG, tendo ficado internada. Houve estabilização clínica e laboratorial com melhoria dos sintomas e teve alta com o provável diagnóstico de SML.

Comentário: Este caso demonstra a importância da monitorização tiroideia e vigilância sintomática dos doentes com patologia tiroideia de base. O médico de família tem um papel fundamental no controlo destas situações por ter um acompanhamento longitudinal dos seus pacientes. Uma abordagem mais abrangente de cada doente e a articulação entre os cuidados de saúde primários e secundários permite a identificação de problemas clínicos específicos e tratamento precoce dos mesmos.

Palavras-chave: Hipertiroidismo; Tirotoxicose; Doença de Graves.

\section{INTRODUÇÃO}

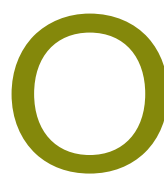

hipertiroidismo (HT) é uma patologia que ocorre devido à síntese e secreção excessivas de hormonas tiroideias pela glândula tiroideia. ${ }^{1-2}$ Muitos autores distinguem HT de tirotoxicose, sendo este último um conceito mais abrangente que inclui, para além de causas endógenas de produção hormonal pela tiroide, causas exógenas. ${ }^{3-5}$

A prevalência do HT é variável, estimando-se que seja entre 1,2 e $1,6 \%$ a nível mundial $-0,5$ a $0,6 \%$ para o HT clínico e 0,7 a $1 \%$ para o HT subclínico. ${ }^{1,3-4,7}$ - e 0,8\% na Europa. ${ }^{8} \mathrm{~A}$ sua prevalência aumenta com a idade e é maior no sexo feminino. ${ }^{7-8}$

A principal etiologia do HT é a doença de Graves (DG), ocorrendo em cerca de $75 \%$ dos casos, ${ }^{5}$ seguida

1. Faculdade de Medicina da Universidade de Lisboa. Lisboa, Portugal. 2. USF Descobertas, ACeS Lisboa Ocidental e Oeiras. Lisboa, Portugal. do bócio multinodular (BMN) tóxico. ${ }^{1-2,7}$ Outras causas menos frequentes correspondem a adenoma da tiroide tóxico, tiroidite ou mais raramente intoxicação medicamentosa. ${ }^{2,4-5,7}$

A DG é uma patologia autoimune por produção de anticorpos antirrecetor da TSH (TRAb) que estimulam o recetor da hormona estimulante tiroideia (TSH) e aumentam a produção endógena e libertação de hormonas tiroideias..$^{1-4}$ É mais frequente no sexo feminino ${ }^{1} \mathrm{e}$ o pico de incidência situa-se entre os 30 e os 60 anos. ${ }^{1,5}$ O BMN tóxico é uma patologia multinodular da glândula tiroide em que os nódulos são hiperfuncionantes e produtores de hormonas tiroideias em excesso, sendo mais comum na população com idade mais avançada, por volta dos 60 anos. É a causa mais frequente de HT nas áreas em que existe défice de iodo. ${ }^{2-5}$

As manifestações clínicas do HT podem variar desde o quadro assintomático até ao quadro de tempestade 
tiroideia, uma situação rara de tirotoxicose aguda grave e potencialmente fatal. ${ }^{2}$ Os principais sintomas são intolerância ao calor, palpitações, tremor, irritabilidade e ansiedade, insónia, fadiga, perda de peso, aumento do apetite e diarreia. ${ }^{2-5}$ Existem outros sintomas, mais específicos da DG, como exoftalmia, edema peri-orbitário, diplopia, mixedema pré-tibial e acropatia tiroideia (edema dos tecidos moles das mãos e hipocratismo digital). ${ }^{2,4}$ Numa situação de HT agudo existe taquicardia com aumento da tensão arterial (TA), cansaço extremo e fraqueza muscular, diaforese, podendo evoluir para tempestade tiroideia caracterizada por febre, fibrilação auricular, insuficiência cardíaca, sintomas neuropsiquiátricos, terminando em coma e morte. ${ }^{3-5,9}$

O diagnóstico do HT é clínico e laboratorial, sendo o quadro clássico caracterizado por uma TSH baixa e tiroxina livre (fT4) e tri-iodotironina livre (fT3) elevadas. ${ }^{1-5}$ A TSH é o marcador mais sensível e específico para diagnóstico de patologia tiroideia. ${ }^{1-2,4} \mathrm{Em}$ alguns casos, a TSH pode estar baixa e a fT4 efT3 encontrarem-se dentro dos valores normais, situação esta que corresponde a um HT subclínico. ${ }^{3-5,7} \mathrm{Na}$ DG, para além das alterações na função tiroideia, os TRAb encontram-se elevados. ${ }^{1-2,5}$ A ecografia da tiroide e posteriormente a cintigrafia de captação de iodo são meios complementares que podem ajudar no diagnóstico do BMN tóxico. ${ }^{2}$ Nos casos de HT agudo, para além do quadro clínico, a TSH encontra-se suprimida e os valores de fT4 e fT3 bastante elevados. ${ }^{9}$

A patologia nodular da tiroide é bastante prevalente, sendo possível detetar por palpação em aproximadamente $5 \%$ das mulheres e $1 \%$ dos homens e detetada em ecografia em 19 a $68 \%$ da população. ${ }^{10}$ No entanto, a maioria desses nódulos não são funcionantes nem causam sintomas. ${ }^{11}$ A coexistência de nódulos tiroideus com a DG pode ocorrer em cerca de $35 \%$ dos casos e entre 0,8 a 2,7\% dos pacientes, os nódulos existentes são hiperfuncionantes, situação essa denominada por síndroma de Marine Lenhart (SML). ${ }^{12-14}$ Esta síndroma rara foi descrita pela primeira vez em $1911^{15}$ e, embora estejam descritos vários casos, ${ }^{16}$ os critérios de diagnóstico são variáveis e ainda não estão bem estabelecidos. ${ }^{13,17}$ Neuman e colaboradores propuseram, em 2018, os seguintes critérios de diagnóstico simplificados: 1) HT com DG comprovado por valores analíticos da função tiroideia e TRAb positivos; 2) aumento da captação de iodo radioativo e presença de nódulos frios e quentes na cintigrafia tiroideia; e 3) biopsia tiroideia revelando lesão hiperplásica ou adenoma folicular. ${ }^{17}$

O caso apresentado descreve uma situação de hipertiroidismo agudo grave numa mulher de 50 anos e pretende elucidar/alertar para a importância da suspeição clínica em pacientes com sintomas inespecíficos.

\section{DESCRIÇÃO DO CASO}

P.B., sexo feminino, 50 anos, natural e residente em Lisboa, solteira, raça caucasiana, empregada de limpeza. Apresenta, como antecedentes pessoais, hipertensão arterial (HTA), medicada com candesartan + hidroclorotiazida $16 \mathrm{mg}+12,5 \mathrm{mg}$ e controlada, dislipidemia medicada com atorvastatina $10 \mathrm{mg}$, estenose aórtica moderada seguida na consulta de cardiologia, excesso de peso [índice de massa corporal (IMC) de $28,7 \mathrm{~kg} / \mathrm{m}^{2}$ ] e HT por BMN desde os 35 anos, seguida em consulta de endocrinologia e medicada com tiamazol até 2018, quando teve alta com recomendação de vigilância anual e de suspensão da medicação por estabilização dos valores tiroideus (entre 2018 e 2020 valores da função tiroideia normais). Como hábitos, a referir tabagismo de 10 cigarros/dia. Nega alergias e apresenta o plano nacional de vacinação (PNV) atualizado. Não apresenta antecedentes familiares relevantes.

Recorre a consulta programada de HTA na unidade de saúde familiar (USF) em 14/07/2020, onde refere maior cansaço para grandes esforços, principalmente no trabalho, sem outra sintomatologia associada. Ao exame objetivo (EO) apresentava peso $74,5 \mathrm{~kg}$, TA 120/60mmHg, frequência cardíaca (FC) de 69bpm, auscultação cardiopulmonar (ACP) com sopro sistólico grau IV/VI audível em todo o precórdio (já conhecido anteriormente) e ausência de edemas dos membros inferiores (MI). Foram pedidas análises de controlo de HTA com função tiroideia e ecografia da tiroide.

Em 29/07/2020 recorre de novo a consulta programada para mostrar o resultado dos exames. A ecografia da tiroide revelou "glândula tiroideia globosa, de contornos bosselados e de dimensões aumentadas com ecoestrutura heterogénea e multinodular com diversos nódulos em ambos os lóbulos". Analiticamente, TSH $0,037 \mu \mathrm{U} / \mathrm{mL}(0,5-4,0 \mu \mathrm{U} / \mathrm{mL})$ e fT4 $25 \mathrm{pmol} / \mathrm{L}$ 
(12- 22pmol/L), sem outras alterações. Mantinha ainda cansaço progressivo para grandes esforços, agora associado a maior irritabilidade e ansiedade, insónia inicial, episódios esporádicos de palpitações e intolerância ao calor. Ao EO apresentava TA 120/68mmHg, FC 93bpm e tiroide palpável de dimensões aumentadas. Foi assumido possível hipertiroidismo descompensado como etiologia das queixas apresentadas e referenciou-se com urgência à consulta de endocrinologia.

Em 24/08/2020, a utente, que ainda não tinha tido consulta de endocrinologia, recorre a consulta aberta na USF por agravamento do quadro clínico, apresentando fraqueza e cansaço extremo com mialgias em ambos os MI com impotência funcional importante para a marcha, insónia grave à três dias, labilidade emocional e irritabilidade associada a palpitações. Ao EO apresenta peso $71 \mathrm{~kg}$ (perda de $3,5 \mathrm{~kg}$ em menos de um mês), TA 110/68mmHg, FC 105bpm com pulso radial rítmico e simétrico, ACP sem alterações de novo, MI sem edemas ou sinais de trombose venosa profunda (TVP), dor na região gemelar bilateral, pior à esquerda e com sinal de Homans positivo à esquerda. Por suspeita de hipertiroidismo agudo e/ou TVP à esquerda referenciou-se ao serviço de urgência (SU).

No SU, no mesmo dia, realizou avaliação analítica que revelou TSH $<0,008 \mu \mathrm{U} / \mathrm{mL}(0,5-4,0 \mu \mathrm{U} / \mathrm{mL})$, fT4 > 100pmol/L (12-22pmol/L), fT3 37,9pmol/L (3,1-6,8pmol/L), TRAb 23,9 U/L ( $<$ 1,58 U/L) e D-dímeros $831 \mathrm{ng} / \mathrm{mL}$ (<500ng/mL), eletrocardiograma (ECG) em ritmo sinusal e com FC de 95bpm e ecodoppler venoso dos MI sem alterações. Foi assumido hipótese diagnóstica de hipertiroidismo grave por doença de Graves, medicou-se com tiamazol $10 \mathrm{mg}$ de $8 / 8 \mathrm{~h}$ e propanolol $40 \mathrm{mg}$ de $12 / 12 \mathrm{~h}$ e a utente ficou internada no serviço de endocrinologia. Durante o internamento foi observada por oftalmologia, que descartou complicações oculares e esteve sempre hemodinamicamente estável, com perfil tensional e FC controlados, verificando-se uma melhoria sintomática e analítica, tendo tido alta em 31/08/2020, com fT4 52pmol/L e fT3 14,3pmol/ /L e com o possível diagnóstico de tireotoxicose por síndroma de Marine Lenhart. Manteve medicação crónica habitual, associando-se tiamazol 10mg de $8 / 8 \mathrm{~h} \mathrm{e}$ propanolol 40mg de 12/12h.

Em 15/09/2020 teve teleconsulta de reavaliação na USF após internamento, referindo melhoria substancial do quadro clínico apesar de ainda manter algum cansaço para grandes esforços e mialgias dos $\mathrm{MI}$, principalmente no trabalho. Em 16/10/2020, na consulta de endocrinologia, realiza nova avaliação analítica que revelou TSH 0,012 U/mL, fT3 8,0pmol/L efT4 9,98pmol/L e ecografia da tiroide, onde mantinha "tiroide aumentada com desvio lateral da traqueia para a esquerda, muito vascularizada e heterogénea com múltiplos nódulos". Clinicamente encontrava-se bem, apresentando apenas ligeiro cansaço para grandes esforços. Fezse ajuste de medicação com redução de tiamazol para $15 \mathrm{mg}$ /dia e foi proposta intervenção cirúrgica com tiroidectomia total que a utente aceitou, ficando a aguardar a mesma.

\section{COMENTÁRIO}

O tratamento do HT consiste no controlo sintomático e no tratamento da etiologia, sendo que o mesmo depende da idade, sintomatologia, comorbilidades e preferência do utente. ${ }^{2}$ Todos os pacientes sintomáticos devem ser tratados com um $\beta$-bloqueante. ${ }^{2-3}$ Relativamente à resolução do HT em si existem três opções terapêuticas - a utilização de fármacos antitiroideus (tiamazol ou propiltiouracil), utilização de iodo radioativo ou ainda a tiroidectomia total, sendo que ambas são eficazes na DG e no BMN tóxico..$^{2-5}$ No SML o tratamento recomendado como primeira linha são os fármacos antitiroideus, podendo ser utilizado iodo radioativo nos casos resistentes ou nos pacientes com importantes efeitos adversos à medicação. Em situações resistentes ao tratamento e nos casos com importante BMN, a cirurgia é a melhor opção. ${ }^{18-19}$ É importante estar alerta para esta situação nos casos de DG, principalmente quando esta requer doses elevadas de medicação ou quando há recorrência da sintomatologia após suspensão dos antitiroideus. ${ }^{19}$

Neste caso em particular optou-se pelo controlo sintomático na fase aguda e tratamento com o antitiroideu tiamazol, com melhoria substancial da função tiroideia e estabilização sintomática.

A recorrência após suspensão de medicação ocorre em 30 a $70 \%$ dos casos de DG, principalmente no primeiro ano após descontinuação, podendo desencadear uma situação de HT agudo que pode ser grave e evoluir para tempestade tiroideia. ${ }^{2}$ A tempestade tiroideia é uma entidade rara, que ocorre em 1 a $5 \%$ dos 
pacientes admitidos no hospital por tirotoxicose com uma taxa de mortalidade entre 8 a $25 \% .{ }^{4}$ Os principais fatores de risco para a sua ocorrência são doença aguda, trauma, cirurgia, stress ou gravidez. ${ }^{4,9}$ Esta situação pode ser distinguida do HT agudo pela existência de disfunção multiorgânica grave, utilizando os critérios de Burch e Wartofsky, que se baseia em parâmetros como a temperatura central, sintomas do sistema nervoso central, sintomas de disfunção gastrointestinal e hepática, a FC, existência de insuficiência cardíaca e fibrilação auricular. ${ }^{4} \mathrm{O}$ tratamento consiste em suporte de vida e controlo da disfunção multiorgânica, bem como do HT. ${ }^{4,9}$

Este caso clínico descrito demonstra uma situação de HT grave, mas sem critérios clínicos de tempestade tiroideia, numa doente com BMN de base não medicada por aparente controlo de função tiroideia. A doente apresentava um quadro clínico típico de HT descompensado, com alguns sintomas que poderiam indicar possível gravidade, como a fraqueza extrema com impotência funcional e a insónia total, mas sem sinais suspeitos de disfunção orgânica grave, como crise hipertensiva, taquicardia, febre ou sinais ou sintomas de insuficiência cardíaca. Analiticamente foi confirmado quadro de HT grave, com valores de hormonas tiroideias muito elevados. Além disso, apresenta critérios de DG, o que a enquadra no diagnóstico possível de SML por possuir antecedentes pessoais de BMN tóxico e ecografia tiroideia recente que demonstra patologia multinodular da tiroide. No entanto, o diagnóstico definitivo só seria possível através de cintigrafia tiroideia com captação de iodo e talvez biopsia, mas tais exames não foram possíveis de realizar. Esta situação de descompensação evoluiu possivelmente de forma progressiva ao longo de meses, com um avanço mais rápido no último mês antes do internamento.

A monitorização da função tiroideia destes pacientes com patologia tiroideia de base é importante, devendo ser realizada a cada um a três meses durante os primeiros seis a doze meses. ${ }^{2}$ Este caso permite salientar também o papel da vigilância sintomática e suspeição precoce do quadro clínico de descompensação, muitas vezes caracterizado por sintomas inespecíficos. A inespecificidade ocorrida nesta situação é um problema em muitas patologias, dificultando o diagnóstico e atrasando o tratamento, por vezes podendo levar a consequências graves. Como tal, o conhecimento dos antecedentes pessoais e a realização de uma boa anamnese e exame objetivo são essenciais na ultrapassagem deste obstáculo.

Em situações como esta, o médico de família (MF) tem um papel fundamental, pois tem um acompanhamento longitudinal dos seus pacientes, prestando cuidados de saúde personalizados ao longo do tempo. Este fator é essencial na abordagem de quadros clínicos inespecíficos e sua orientação, como acabou por ocorrer no caso descrito. Além disso, é responsável pelo acompanhamento mais regular da maioria das patologias crónicas, contribuindo para o seu controlo. O MF também gere situações agudas, muitas vezes evitando idas desnecessárias ao SU, avaliando a real necessidade da articulação com o mesmo. A abordagem abrangente de cada paciente, tendo em conta os seus vários contextos, também traz vantagens na identificação de problemas clínicos específicos e na sua gestão, contribuindo para o envolvimento de cada doente na sua saúde.

Salienta-se igualmente a importância dos cuidados de saúde primários como porta de entrada principal do sistema de saúde, sendo fundamental uma boa acessibilidade para o funcionamento do mesmo, facto este demonstrado no presente caso - a utente recorreu precocemente à USF, tendo-lhe sido prestada uma resposta atempada e eficaz.

Por fim, este caso demonstra também a importância da articulação entre os cuidados de saúde primários e secundários na gestão de patologias agudas e crónicas, facilitando o diagnóstico e tratamento precoce e eficaz das mesmas.

\section{REFERÊNCIAS BIBLIOGRÁFICAS}

1. Kahaly GJ, Bartalena L, Hegedüs L, Leenhardt L, Poppe K, Pearce SH. 2018 European Thyroid Association guideline for the management of Graves' hyperthyroidism. Eur Thyroid J. 2018;7(4):167-86.

2. Kravets I. Hyperthyroidism: diagnosis and treatment. Am Fam Physician. 2016;93(5):363-70.

3. Ross DS, Burch HB, Cooper DS, Greenlee MC, Laurberg P, Maia AL, et al. 2016 American Thyroid Association guidelines for diagnosis and management of hyperthyroidism and other causes of thyrotoxicosis. Thyroid. 2016;26(10):1343-421.

4. De Leo S, Lee SY, Braverman LE. Hyperthyroidism. Lancet. 2016;388 (10047):906-18.

5. Vaidya B, Pearce SH. Diagnosis and management of thyrotoxicosis. BMJ. 2014;349:g5128. 
6. Taylor PN, Albrecht D, Scholz A, Gutierrez-Buey G, Lazarus JH, Dayan $\mathrm{CM}$, et al. Global epidemiology of hyperthyroidism and hypothyroidism. Nature Rev Endocrinol. 2018;14(5):301-16.

7. Vanderpump MP. The epidemiology of thyroid disease. Br Med Bull. 2011;99:39-51.

8. Garmendia-Madariaga A, Santos-Palacios S, Guillén-Grima F, Galofré JC. The incidence and prevalence of thyroid dysfunction in Europe: a meta-analysis. J Clin Endocrinol Metab. 2014;99(3):923-31.

9. Idrose AM. Acute and emergency care for thyrotoxicosis and thyroid storm. Acute Med Surg. 2015;2(3):147-57.

10. Haugen BR, Alexander EK, Bible KC, Doherty GM, Mandel SJ, Nikiforov YE, et al. 2015 American Thyroid Association management guidelines for adult patients with thyroid nodules and differentiated thyroid cancer: the American Thyroid Association Guidelines Task Force on Thyroid Nodules and Differentiated Thyroid Cancer. Thyroid. 2016;26(1):1-133;

11. Paschou SA, Vryonidou A, Goulis DG. Thyroid nodules: a guide to assessment, treatment and follow-up. Maturitas. 2017;96:1-9.

12. Kim WB, Han SM, Kim TY, Nam-Goong IS, Gong G, Lee HK, et al. Ultrasonographic screening for detection of thyroid cancer in patients with Graves' disease. Clin Endocrinol. 2004;60(6):719-25.

13. Charkes ND. Graves' disease with functioning nodules (Marine-Lenhart syndrome). J Nucl Med. 1972;13(12):885-92.

14. Carnell NE, ValenteWA. Thyroid nodules in Graves' disease: classification, characterization, and response to treatment. Thyroid. 1998;8(8):647-52.
15. Marine D, Lenhart C. Pathological anatomy of exophthalmic goitre. Arch Intern Med. 1911;8:265-316.

16. Biersack HJ, Biermann K. The Marine-Lenhart syndrome revisited. Wien Klin Wochenschr. 2011;123(15-16):459-62.

17. Neuman D, Kuker R, Vendrame F. Marine-Lenhart syndrome: case report, diagnosis, and management. Case Rep Endocrinol. 2018;2018: 3268010.

18. Maurício J, Rocha MA, Heitor S. Marine-Lenhart syndrome: a rare cause of hyperthyroidism. Rev Port Endocrinol Diabetes Metab. 2020;15(12):89-91.

19. Cakir M. Marine-Lenhart syndrome. J Natl Med Assoc. 2005;97(7):10368.

\section{CONFLITO DE INTERESSES}

Os autores declaram não possuir quaisquer conflitos de interesse.

\author{
ENDEREÇO PARA CORRESPONDÊNCIA \\ Valter Filipe Moreira \\ E-mail: valtermoreira91@gmail.com \\ https://orcid.org/0000-0002-8426-2985
}

Recebido em 28-10-2020

Aceite para publicação em 25-05-2021

\section{ABSTRACT}

\section{ACUTE HYPERTHYROIDISM: A LIKELY MARINE LENHART SYNDROME'S CASE REPORT}

Introduction: Hyperthyroidism (HT) is a pathology that occurs due to the synthesis and excessive secretion of thyroid hormones by the thyroid gland and its prevalence worldwide is between 1.2 to $1.6 \%$. The main etiology of HT is Graves' disease (GD), followed by toxic multinodular goiter (MNG). The clinical manifestations of HT can vary from asymptomatic to a thyroid storm, a rare situation of severe acute and potentially fatal thyrotoxicosis. Marine Lenhart syndrome (MLS) consists of an HT that coexists with a toxic MNG and GD. It is a rare situation that occurs in 0.8 to $2.7 \%$ of patients with GD.

Case description: A 50-year-old woman, with a personal history of unmedicated toxic, MNG, goes to an appointment due to an extreme weakness with functional impotence for walking, severe insomnia, emotional lability, and irritability associated with palpitations. She was referred to the emergency department where she was diagnosed with severe HT due to GD, having been hospitalized. There was clinical and laboratory stabilization with an improvement of symptoms, and she was discharged from the hospital with the probable diagnosis of MLS.

Comments: This case demonstrates the importance of thyroid monitoring and supervision of symptoms in patients with thyroid disease. The family doctor has a fundamental role in controlling these situations because of the longitudinal monitoring of their patients. A broader approach to each patient and the link between primary and secondary health care allow the identification of specific clinical problems and their early treatment.

Keywords: Hyperthyroidism; Thyrotoxicosis; Graves' disease. 